\title{
Processing of GRAS/METOP radio occultation data recorded in closed-loop and raw-sampling modes
}

\author{
M. E. Gorbunov ${ }^{1,2}$, K. B. Lauritsen ${ }^{2}$, H.-H. Benzon ${ }^{2}$, G. B. Larsen ${ }^{2}$, S. Syndergaard ${ }^{2}$, and M. B. Sørensen ${ }^{2}$ \\ ${ }^{1}$ A. M. Obukhov Institute for Atmospheric Physics, Russian Academy of Sciences, Moscow, Russia \\ ${ }^{2}$ Danish Meteorological Institute, Copenhagen, Denmark
}

Received: 3 February 2011 - Published in Atmos. Meas. Tech. Discuss.: 15 February 2011

Revised: 9 May 2011 - Accepted: 26 May 2011 - Published: 9 June 2011

\begin{abstract}
Instrument GRAS (Global Navigation Satellite System Receiver for Atmospheric Sounding) on-board of the Metop-A satellite was activated on 27 October 2006. Currently, Metop-A is a fully operational satellite with GRAS providing from 650-700 occultations per day. We describe our processing of GRAS data based on the modification of our OCC software, which was modified to become capable of reading and processing GRAS data. We perform a statistical comparison of bending angles and refractivities derived from GRAS data with those derived from ECMWF analyses. We conclude that GRAS data have error characteristics close to those of COSMIC data. In the height range $10-30 \mathrm{~km}$, the systematic refractivity difference GRAS-ECMWF is of the order of $0.1-0.2 \%$, and the standard deviation is $0.3-$ $0.6 \%$. In the lower troposphere GRAS refractivity and bending angle indicate a negative bias, which reaches its maximum value in the tropics. In particular the retrieved refractivity is biased by up to $2.5 \%$. The negative bias pattern is similar to that found in the statistical validation of COSMIC data. This makes it probable that the bias should not be attributed to the instrument design or hardware.
\end{abstract}

\section{Introduction}

The instrument GRAS (Global Navigation Satellite System Receiver for Atmospheric Sounding) is a new generation instrument for the radio occultation (RO) sounding of the Earth's atmosphere. The instrument was launched by EUMETSAT (European Organization for the Exploitation of Meteorological Satellites) on-board of the Metop-A satellite on 19 October 2006 and activated on 27 October 2006 (von

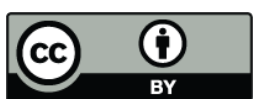

Correspondence to: M. E. Gorbunov (m_e_gorbunov@mail.ru)
Engeln et al., 2009; Bonnedal et al., 2010a,b). GRAS was designed for observing setting and rising occultations from the GPS (Global Positioning System) satellite constellation. Measurements are performed in closed-loop (CL) and rawsampling (RS) modes (Luntama et al., 2008; Bonnedal et al., 2010a,b). From the processing view point, these modes are similar to phase-locked loop (PLL) and open-loop (OL) modes implemented in Constellation Observing System for Meteorology, Ionosphere, and Climate (COSMIC) (Rocken et al., 2000; Sokolovskiy et al., 2009).

Both in COSMIC and in GRAS, CL measurements are performed with $50 \mathrm{~Hz}$ sampling rate. CL mode is suitable for measurements with weak fluctuations of the phase. In processing of CHAMP and COSMIC data it was found that CL mode provides a good quality of measurements above $7-11 \mathrm{~km}$ in the tropics (Gorbunov and Kornblueh, 2003; Sokolovskiy et al., 2009). In the lower troposphere in the tropics the signal undergoes strong scintillations of both amplitude and phase, which degrades the quality of signal tracking and results in the increase of retrieval errors. This effect is weaker in middle latitudes. In polar latitudes, it is possible to perform retrievals using the CL mode down to the Earth's surface. In the CL mode, the receiver typically uses a two-quadrant detector, which is insensitive to the sign of the signal (or to the change of the phase by $\pi$ radian) (Beyerle et al., 2003). This allows for automatically removing the signal modulation by the navigation bits, but results in the loss of half-cycles if the phase variations between samples exceed $\pi / 2$ radian.

Measurements in the OL mode in COSMIC are also performed at a sampling rate of $50 \mathrm{~Hz}$. In the OL mode, the signal frequency is down-converted by an oscillator numerically controlled by a phase model independent from the measured signal, i.e. without the feed-back. Because it is always possible to provide a phase model predicting the Doppler frequency of the signal with an accuracy of $10-15 \mathrm{~Hz}$, the

Published by Copernicus Publications on behalf of the European Geosciences Union. 


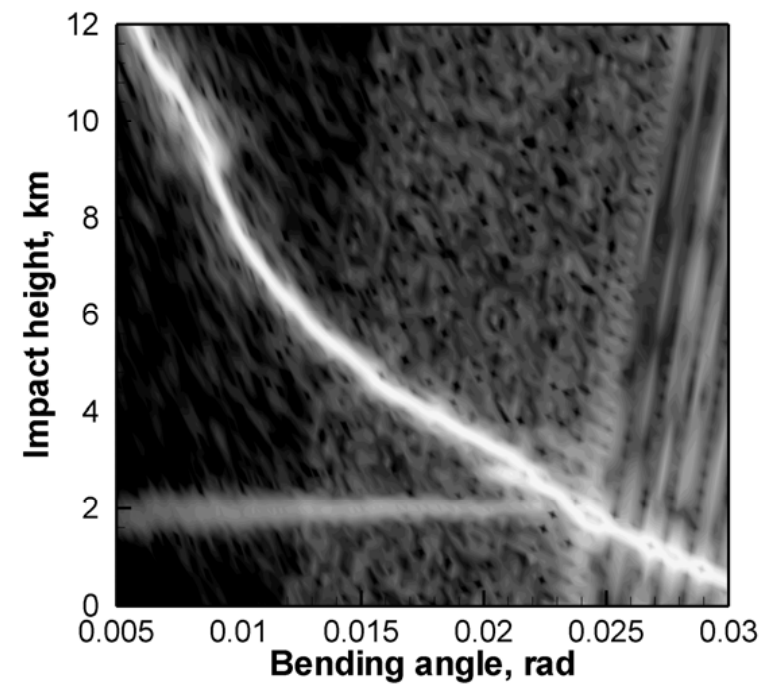

Fig. 1. Occultation event observed on 30 September 2007, UTC 01:22, 56.72S 3.53E. Reflection and multipath over Atlantic Ocean.

$50 \mathrm{~Hz}$ sampling rate is sufficient for the correct retrieval of the signal phase (Sokolovskiy et al., 2009). We use a phase model based on the MSIS climatology (Hedin, 1991) complemented with a constant relative humidity of $80 \%$ below the height of $15 \mathrm{~km}$. Measurements in the OL mode are performed by a four-quadrant detector, which can measure phase in the full range from 0 to $2 \pi$ radian. However the removal of the navigation bits becomes a problem, which is resolved by collecting them from ground-based stations (Sokolovskiy et al., 2009). This is termed external demodulation. Another possibility is to use an internal demodulation algorithm, which is based on the identification of the phase jumps by $\pi$ radian. The internal demodulation only works if the phase variation between the samples does not exceed $\pi / 2$ radian. Otherwise it results in the loss of half-cycles and the degradation of the retrieval quality (Sokolovskiy et al., 2009).

Unlike the OL mode in COSMIC, the RS mode in GRAS employs a $1 \mathrm{kHz}$ sampling rate. This results in much smaller variations of the phase between signal samples as compared to $50 \mathrm{~Hz}$ sampling. The signal phase variation for $1 \mathrm{kHz}$ sampling is small enough to perform the internal demodulation without employing externally supplied navigation bits. There is, however, a trade-off between the decreased phase variation and the increased additive noise level which for $1 \mathrm{kHz}$ is $\sqrt{20}$ times greater than it is for $50 \mathrm{~Hz}$. The $50 \mathrm{~Hz}$ sampling rate is insufficient for the correct internal demodulation of signals from the lower troposphere in the tropics (Sokolovskiy et al., 2009). On the other hand, $1 \mathrm{kHz}$ may be too high. The choice of the optimal sampling rate should be addressed in the future research.

In this paper we describe our processing of offline GRAS data and present the statistical comparisons of bending angles

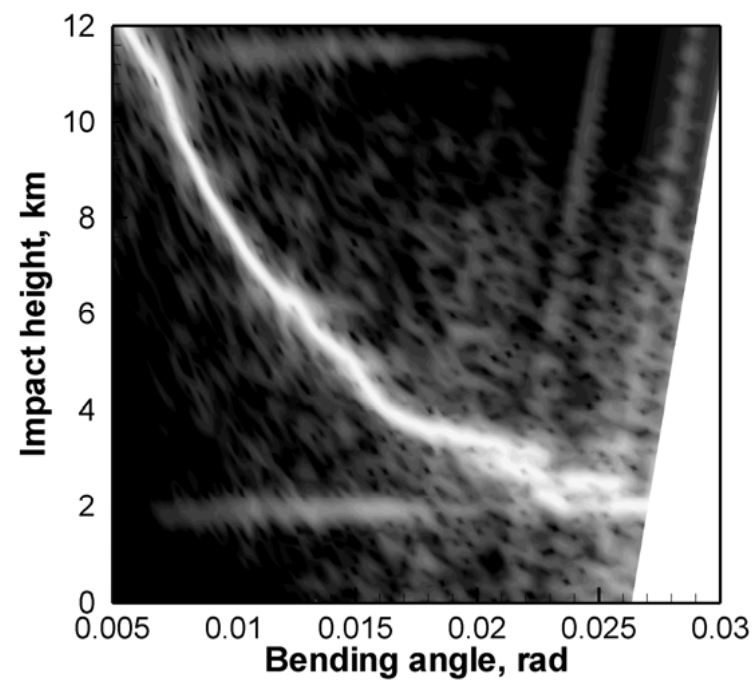

Fig. 2. Occultation event observed on 30 September 2007, UTC 21:47, 65.93S 87.20E. Reflection and multipath near the Antarctic.

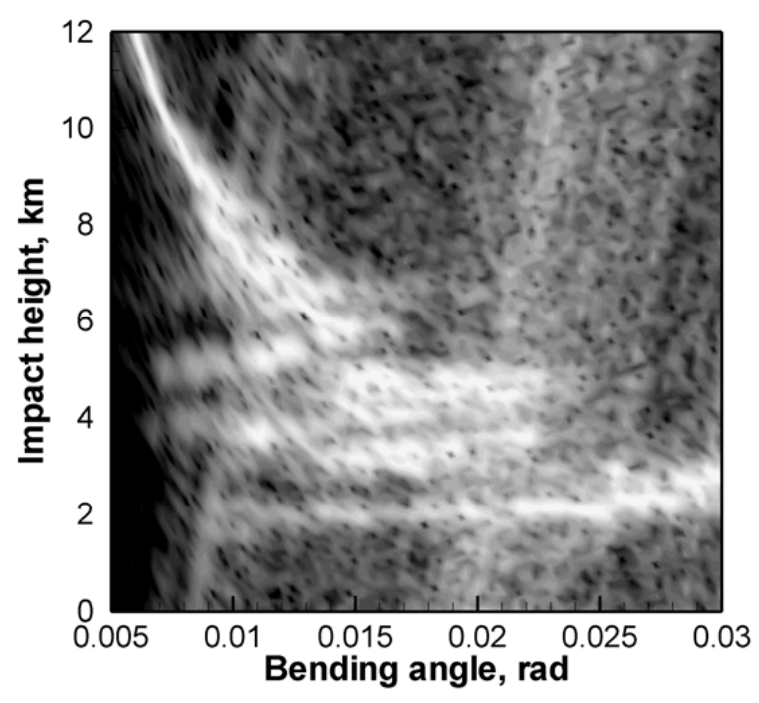

Fig. 3. Occultation event observed on 30 September 2007, UTC $12: 42,45.37 \mathrm{~N} 29.34 \mathrm{~W}$. Strong multipath over Atlantic Ocean.

and refractivities retrieved from GRAS observations with the ECMWF analyses. We show that GRAS data have a quality comparable to that of COSMIC data.

\section{The data processing}

For processing GRAS data we modified the OCC software (Gorbunov et al., 2011). We added modules for the automatic recognition and reading of GRAS data files. As the first step the following variables are obtained from the files: the GPS and Metop coordinates in the Earth's centered inertial frame, amplitudes and phase excesses for L1 and L2, and navigation bits. The satellite coordinates, amplitudes, 

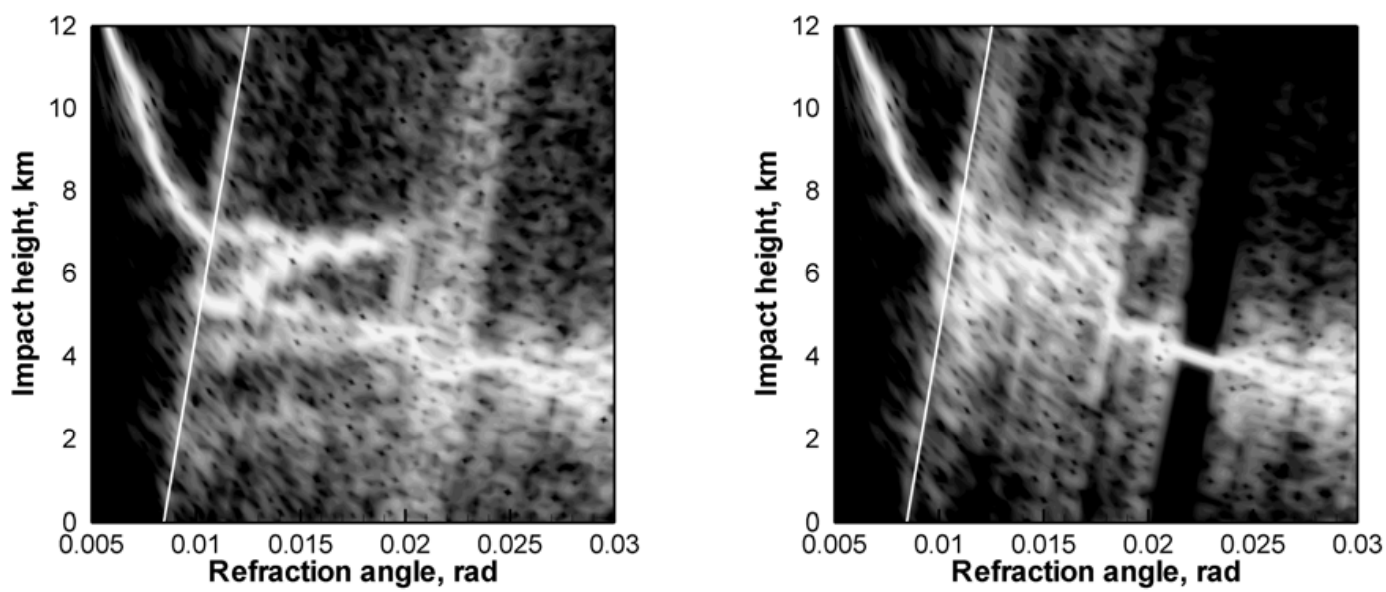

Fig. 4. Occultation event observed on 30 September 2007, UTC 00:12, 4.67S 25.31W. Left panel: merging mode RS+CL. Right panel: CL data only. The white line shows the border between the CL area and the RS + CL area.

and phase excesses are organized as two separate records: $\mathrm{CL}$ and RS. Navigation bits are only recorded for RS mode. The files contain two types of navigation bits: external, obtained from the ground-based stations, and internal, obtained from the phase by identifying the phase jumps by $\pi$ radian between samples.

In most cases, CL and RS records overlap. When RS mode is active, both RS and CL records only contain L1 data. We implemented two modes of merging CL and RS data: (1) use CL data only, (2) use the complete RS record complemented with CL data where no RS data are present (RS+CL). Below we present a comparison of refractivity retrievals for both merging modes. The merged data are re-sampled with a uniform sampling rate, which can be specified by the user. For example, if in the RS+CL merging mode $1 \mathrm{kHz}$ re-sampling is requested, then the RS data keep their full sampling rate, and the CL data are up-sampled at $1 \mathrm{kHz}$.

GRAS/METOP data contain a significant amount of data gaps both in CL and RS modes (Bonnedal et al., 2010a,b). Gaps in CL mode mostly occur when RS data are present. For each gap its length is evaluated. If the gap length exceeds some pre-specified threshold the data after the gap for setting events or, correspondingly, the data before the gap for rising events are discarded. If the gap lenght does not exceed the threshold, the deviation of the phase excess from the phase model and the amplitude inside the gap are linearly interpolated to the uniform time grid between the two surrounding points where the signal is present. This fill-in procedure introduces some additional uncertainty. However, if the gap length threshold is chosen small enough, the uncertainty will also be insignificant. In this study the threshold was $0.04 \mathrm{~s}$.

The data processing algorithm follows the guidelines described in (Gorbunov et al., 2006, 2011). The main steps include: (1) quality control and extrapolation of missing L2 where RS mode is active, (2) combination of bending (refraction) angle retrieval based on geometric optics (GO) above
$25 \mathrm{~km}$ and wave optics below $25 \mathrm{~km}$ using the CT2 algorithm introduced by Gorbunov and Lauritsen (2004), the lowest altitude of a retrieved bending angle profile is determined as the maximum of the correlation of the CT2 amplitude with the step function (Gorbunov et al., 2006), (3) ionospheric correction combined with the statistical optimization (Gorbunov, 2002a), (4) standard refractivity retrieval by the Abel inversion, and (5) dry temperature retrieval.

\section{Results}

Figure 1 shows an example of the spectrogram of RO data (Hocke et al., 1999; Gorbunov, 2002b) indicating a reflection from the ocean surface combined with minor atmospheric multipath propagation effects. Reflected rays form the almost horizontal branch of the bending angle profile near the impact height of $2 \mathrm{~km}$. The bending angle profile below $2 \mathrm{~km}$ is not related to the atmosphere, because below $2 \mathrm{~km}$ there are no rays reaching the receiver. The profile here is obtained from the phase model used to fill in the area where the receiver was unable to track the signal. This part of the profile is discarded in the inversion. Multipath propagation results from a non-monotonic bending angle profile near the impact height of $2.7 \mathrm{~km}$. Figure 2 shows a similar example where reflection is combined with stronger multipath effects. Figure 3 shows an example with very strong multipath effects resulting in a wide-band signal. These selected cases demonstrate the ability of the GRAS instrument to correctly measure RO signals in presence of multipath induced both by the atmospheric conditions and the Earth's surface reflections.

Figure 4 shows an example of tropical event with strong multipath. This figure also illustrates the merging of CL and RS data. The left panel shows the spectrogram for RS+CL merging mode, the right panel shows the spectrum for $\mathrm{CL}$ data only. The white line shows the border between the area where only CL data are present and the area where RS mode 

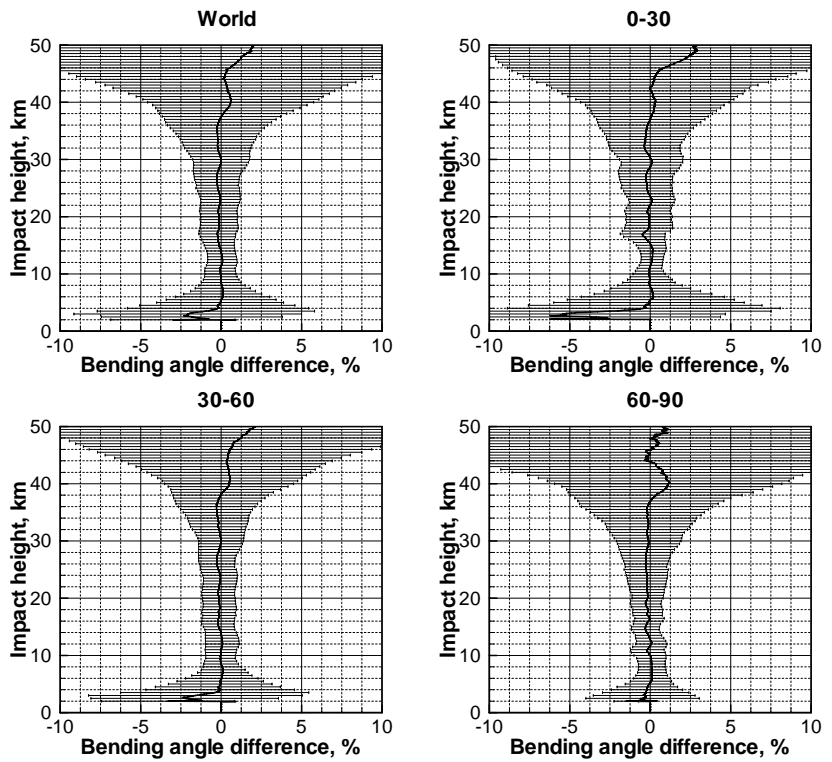

Fig. 5. Statistical comparison of GRAS RS+CL raw bending angles for October 2007 with bending angles obtained by the forward modeling from ECMWF analyses: the whole globe ("World"), tropics (“0-30”), mid-latitudes (“30-60”), and polar latitudes (“60-90”).

is activated and both CL and RS data are available. The multipath structure is very distinct for the RS data. For the CL data the spectrogram is slurred, it only allows for tracing the outlines of the multipath area.

Figure 5 shows the statistical comparison of raw bending angles obtained from offline GRAS data using the RS+CL merging mode with the bending angles obtained by means of the GO forward modeling from the analyses of the European Center for Medium-Range Weather Forecast (ECMWF). Raw bending angles are defined as the linear combination of L1 and L2 bending angles (Vorob'ev and Krasil'nikova, 1994) without statistical optimization. Raw bending angles are independent from any background information. The comparison is based on $14250 \mathrm{RO}$ events observed during September 30 and 1-27 October 2007. The systematic difference GRAS-ECMWF does not exceed $0.3 \%$ in the height range $6-38 \mathrm{~km}$ in all latitude bands, with one exception: Around $16 \mathrm{~km}$ in the tropics it reaches $0.5 \%$. This behavior is explained by the sharp tropopauses unresolved by the filter window of $2 \mathrm{~km}$ used in our data processing, while the ECMWF analyses have a higher resolution of $0.3-$ $0.5 \mathrm{~km}$ here. Larger biases above $38-44 \mathrm{~km}$ are attributed to ECMWF analyses. Bending angles below $4-5 \mathrm{~km}$ are characterized by a negative bias (Sokolovskiy et al., 2010; Gorbunov et al., 2010, 2011; Marquardt et al., 2010, Lauritsen et al., 2010) which has the highest magnitude reaching $6 \%$ in the tropics. The mid-latitudes indicate a moderate negative bias of $3 \%$, in the polar region it almost disappears. Possible mechanisms responsible for the negative bias were discussed by Sokolovskiy et al. (2010) and Gorbunov et al. (2010).
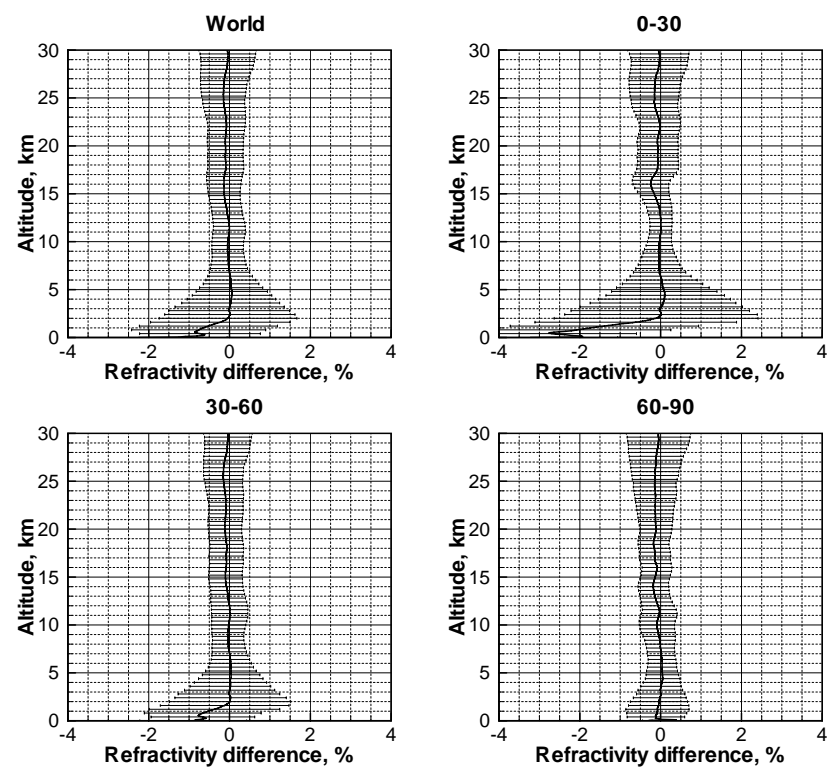

Fig. 6. Statistical comparison of GRAS RS+CL and ECMWF refractivities for October 2007: the whole globe ("World"), tropics (“0-30"), mid-latitudes ("30-60"), and polar latitudes (“60-90”).

Figure 6 shows the statistical comparison of refractivities retrieved from GRAS (RS+CL) data with the ECMWF analyses. The comparison is shown in the height range of 0 $30 \mathrm{~km}$. Above $35-45 \mathrm{~km}$ the residual error of the ionospheric correction becomes comparable with the weather variations of refractivity. The statistically-optimal use of the background atmospheric model at large heights where the signal is noisy (Gorbunov, 2002a) is referred to as the initialization. In particular, in this study we were using bending angle profiles computed from the MSIS climatology and subjected to two-parameter fitting (Lohmann, 2007). Below about $30 \mathrm{~km}$ retrieved refractivities are almost insensitive to the initialization. The systematic differences and standard deviations of GRAS-retrieved refractivities from the ECMWF analyses are very close to similar characteristics of COSMIC retrievals (Gorbunov et al., 2011; Marquardt et al., 2010; Lauritsen et al., 2010). The results presented in Figs. 5 and 6 were obtained for the external navigation bits removal. We have performed a similar analysis with internal navigation bits removal where we find that the statistics are having negligible difference from these figures.

Figure 7 shows the statistical comparison of refractivities retrieved from GRAS CL data with the ECMWF analyses. The comparison is based on $506 \mathrm{RO}$ events observed during 30 September 2007, in particular there are 183 tropical events. 

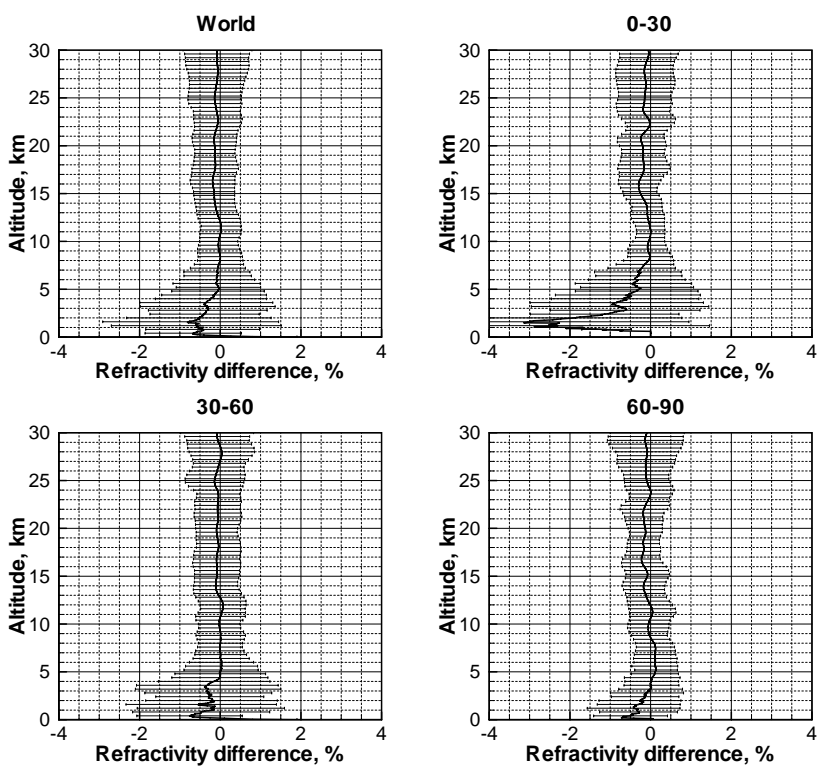

Fig. 7. Statistical comparison of GRAS CL and ECMWF refractivities for 30 September 2007: the whole globe ("World"), tropics (“0-30"), mid-latitudes (“30-60"), and polar latitudes ("60-90”).

Figure 8 shows the penetration. The CL data does not penetrate as deep as the RS data. In the tropics, a negative bias is observed below $8 \mathrm{~km}$, while RS data only indicate a negative bias below $2 \mathrm{~km}$. In the mid-latitudes the bias is observed below a height of $4.5 \mathrm{~km}$, and in the polar latitudes it is observed below $3 \mathrm{~km}$. In the polar latitudes, the bias has a magnitude of $0.5 \%$, which significantly exceeds the value of around $0.1 \%$ obtained for RS data.

\section{Conclusions}

GRAS is a modern instrument for RO measurements. For sounding the lower troposphere raw-sampling (RS) mode is implemented, which allows for measurements of wave fields with strong multipath effects. The $1 \mathrm{kHz}$ sampling rate activated in the RS mode allows for the accurate removal of the navigation message even in the absence of externally supplied navigation bits. The error characteristics of retrieved refractivity are similar to those of COSMIC measurements. The retrievals indicate the same pattern of a negative bias in the lower troposphere. Because two different instruments have similar negative bias, it is most probable that the bias should not be attributed to the instrument design or hardware. Further research should concentrate on the mechanisms resulting in the negative bias discussed by Sokolovskiy et al. (2010) and Gorbunov et al. (2010): the negative bias may result from the systematic loss of large bending angle peaks due to cut-off, contamination with noise and impact parameter variations along the ray path resulting from horizontal gradients of refractivity.
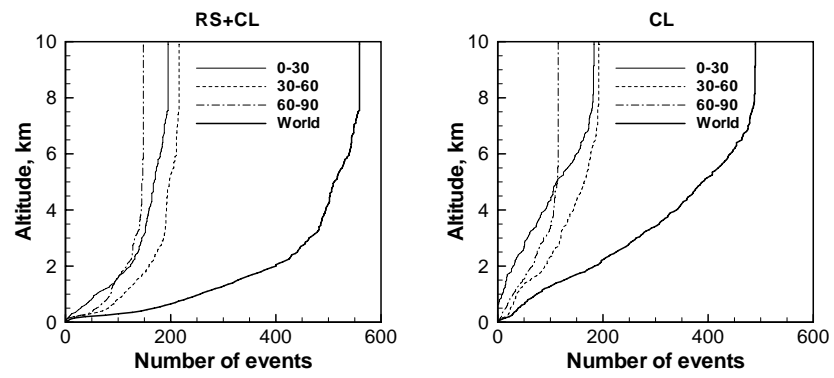

Fig. 8. Penetration depth for RO events observed on 30 September 2007 and processed in the RS+CL (left panel) and CL (right panel) merging modes: the whole globe ("World"), tropics ("030"), mid-latitudes (“30-60”), and polar latitudes (“60-90”).

Acknowledgements. The offline GRAS data used in this study was provided by EUMETSAT and the analysis fields by ECMWF. We appreciate the discussions with Magnus Bonnedal, Christian Marquardt, and the whole GRAS RO Performance Study team represented by RUAG Space, EUMETSAT, Wegener Center at University of Graz, and GFZ Potsdam. This work was performed under ESA contract no. 21995/08/NL/EL. M.E.G. was also supported by the Russian Foundation for Basic Research (grant No. 09-05-00180-a). K.B.L. and S.S. were also supported by the GRAS Satellite Application Facility, which is a decentralized processing center under EUMETSAT.

Edited by: U. Foelsche

\section{References}

Beyerle, G., Gorbunov, M. E., and Ao, C. O.: Simulation Studies of GPS Radio Occultation Measurements, Radio Sci., 38(5), 1084, doi:10.1029/2002RS002800, 2003.

Bonnedal, M., Christensen, J., Carlström, A., and Berg, A.: MetopGRAS in-Orbit Instrument Performance, GPS Solutions 14 pp., 109-120, 2010a.

Bonnedal, M., Carlström, A., Christensen, J., and Lindgren, T.: Instrument Characteristics and Performance Evaluation, Presentation at Presentation at the OPAC 2010 Int. Workshop, Wegener Center, Graz, Austria, 6-10 September 2010b.

Gorbunov, M. E.: Ionospheric correction and statistical optimization of radio occultation data, Radio Science, 37(5), 17-1-17-9, doi:10.1029/2000RS002370, 2002a.

Gorbunov, M. E.: Radioholographic analysis of radio occultation data in multipath zones, Radio Science, 37(1), 14-1-14-9, doi:10.1029/2000RS002577, 2002b.

Gorbunov, M. E. and Kornblueh, L.: Analysis and validation of Challenging Minisatellite Payload (CHAMP) radio occultation data, J. Geophys. Res., 108(D18), 4584, doi:10.1029/2002JD003175, 2003.

Gorbunov, M. E. and Lauritsen, K. B., Analysis of wave fields by Fourier Integral Operators and its application for radio occultations, Radio Science, 39(4), RS4010, doi:10.1029/2003RS002971, 2004.

Gorbunov, M. E., Lauritsen, K. B., Rhodin, A., Tomassini, M., and Kornblueh, L.: Radio holographic filtering, error estimation, 
and quality control of radio occultation data, J. Geophys. Res., 111(D10), D10105, doi:10.1029/2005JD006427, 2006.

Gorbunov, M. E., Lauritsen, K. B., and Leroy, S. S.: Application of Wigner distribution function for analysis of radio occultations, Radio Science, 45, RS6011, doi:10.1029/2010RS004388, 2010.

Gorbunov, M. E., Shmakov, A. V., Leroy S. S., and Lauritsen, K. B.: COSMIC radio occultation processing: Cross-center comparison and validation, J. Atmos. Oceanic Tech., submitted, 2011.

Hedin, A. E.: Extension of the MSIS Thermosphere Model into the Middle and Lower Atmosphere, J. Geophys. Res., 96(A2), 1159-1172, doi:10.1029/90JA02125, 1991.

Hocke, K., Pavelyev, A. G., Yakovlev, O. I., Barthes, L., and Jakowski, N.: Radio Occultation Data Analysis by the Radioholographic Method, Journal of Atmospheric and SolarTerrestrial Physics, 61(15), 1169-1177, 1999.

Lauritsen, K. B., Gorbunov, M. E., Marquardt, C., Bonnedal, M., Benzon, H.-H., Larsen G. B., Syndergaard, S., Sørensen, M. B., and the GRAS raw sampling study team: Processing of GRAS Raw Sampling Data, Presentation at the OPAC 2010 Int. Workshop, Wegener Center, Graz, Austria, 6-10 September 2010.

Lohmann, M. S.: Analysis of global positioning system (GPS) radio occultation measurement errors based on Satellite de Aplicaciones Cientificas-C (SAC-C) GPS radio occultation data recorded in open-loop and phase-locked-loop mode, J. of Geophys. Res., 112, D09115, doi:10.1029/2006JD007764, 2007.

Luntama, J.-P., Kirchengast, G., Borsche, M., Foelsche, U., Steiner, A., Healy, S., von Engeln, A., O'Clerigh, E., and Marquardt, C.: Prospects of the EPS GRAS mission for operational atmospheric applications, Bull. Amer. Meteor. Soc., 89(12), 18631875, 2008.
Marquardt, C. and the GRAS Raw Sampling Study Team: Overview on GRAS - raw sampling and data quality aspects, Presentation at the OPAC 2010 Int. Workshop, Wegener Center, Graz, Austria, 6-10 September 2010.

Rocken, C., Kuo, Y.-H., Schreiner, W. S., Hunt, D., Sokolovskiy, S., McCormick, Ch.: COSMIC System Description, Terrestrial, Atmospheric and Oceanic Science, 11(1), 21-52, 2000.

Sokolovskiy, S., Rocken, C., Schreiner, W., Hunt, D., and Johnson, J.: Postprocessing of L1 GPS Radio Occultation Signals Recorded in Open-Loop Mode, Radio Science, 44, RS2002, doi:10.1029/2008RS003907, 2009.

Sokolovskiy, S., Rocken, C., Schreiner, W., and Hunt, D.: On the Uncertainty of Radio Occultation Inversions in the Lower, J. Geophys. Res.-Atmos., 115, D22111, doi:10.1029/2010JD014058, 2010.

von Engeln, A., Healy, S., Marquardt, C., Andres, Y., and Sancho, F.: Validation of operational GRAS Radio Occultation Data, Geophys. Res. Lett., 36, L17809, doi:10.1029/2009GL039968, 2009.

Vorob'ev, V. V. and Krasil'nikova, T. G.: Estimation of the Accuracy of the Atmospheric Refractive Index Recovery from Doppler Shift Measurements at Frequencies Used in the NAVSTAR System, Izvestiya Academy of Sciences SSSR, Atmospheric and Oceanic Physics, English Translation, 29(5), 602609, 1994. 